\title{
Identification and localization of
}

cholinergic nerve endings and acetylcholine receptors in tissues of the immune system= animal and post-mortem human study
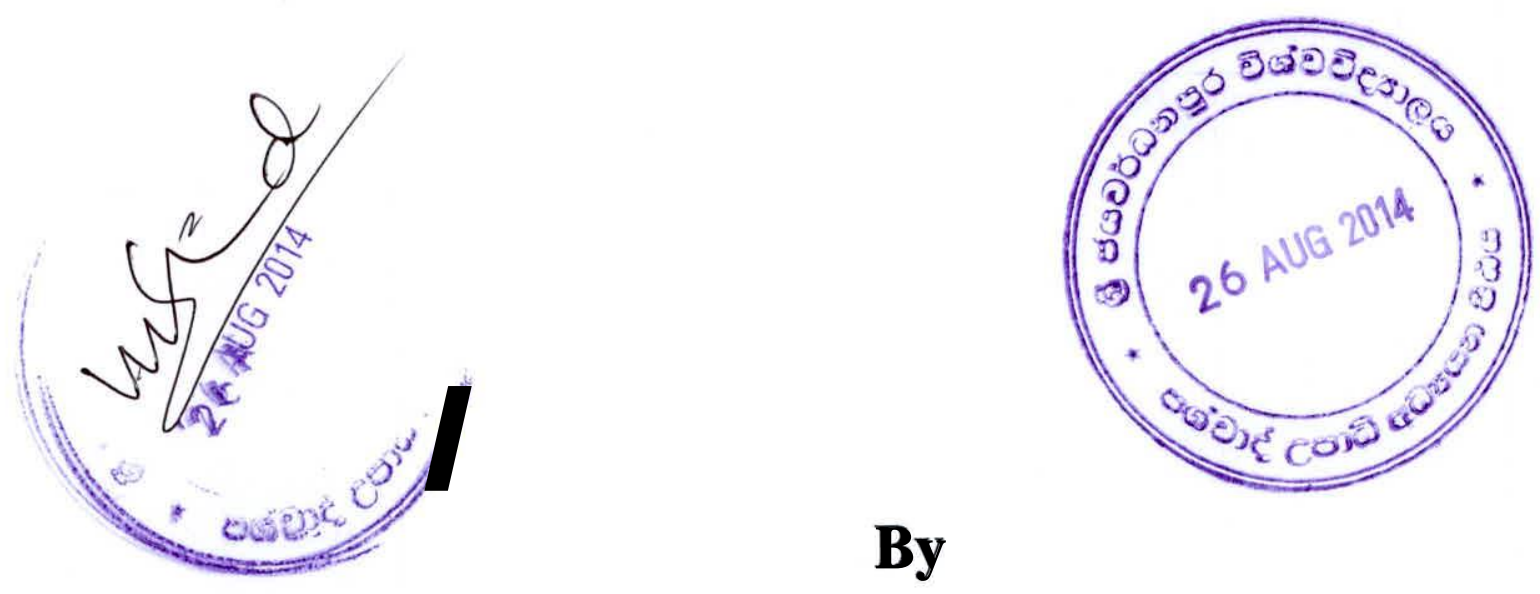

By

Mythreye Thayabaran 


\section{Identification and localization of cholinergic}

nerve endings and acetylcholine receptors in tissues of the immune system- animal and post-mortem human study

By

Mythreye Thayabaran

Thesis submitted to the University of

Sri Jayewardenepura for the award of the Degree of Doctor of Philosophy in Anatomy on the $23^{\text {rd }}$ of May, 2013 


\section{Declaration by the candidate}

The work described in this thesis was carried out by me under the supervision of,

Professor Surangi Gayaneetha Yasawardene

Professor of Anatomy,

Department of Anatomy,

Faculty of Medical Sciences,

University of Sri Jayewardenepura.

A report on this has not been submitted in whole or in part to any university or any other institution for another Degree or Diploma.

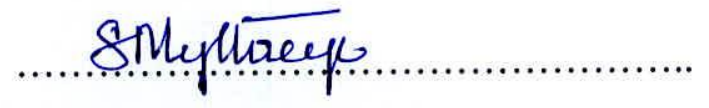

Mythreye Thayabaran
$26.08 \cdot 2014$

Date 


\section{Declaration by the Supervisor}

I certify that the above statement made by the candidate is true and that this thesis is suitable for submission to the University of Sri Jayewardenepura for the purpose of evaluation.

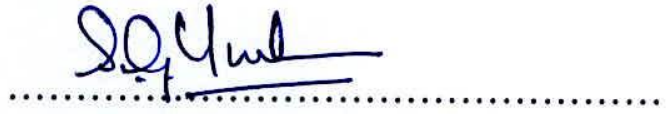

Professor Surangi G.Yasawardene

Head and internal Supervisor,

Department of Anatomy,

Faculty of Medical Sciences,

University of Sri Jayewardenepura. 
I certify that the candidate has incorporated all corrections, additions and amendments recommended by the examiners.

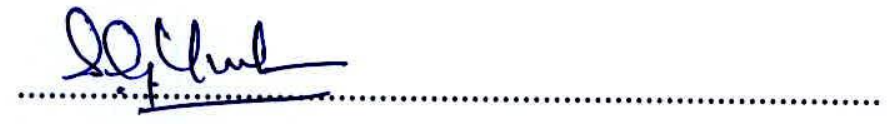

Prof. Surangi G.Yasawardene

Head and internal Supervisor,

Department of Anatomy,

Faculty of Medical Sciences,

University of Sri Jayewardenepura. 


\section{TABLE OF CONTENTS}

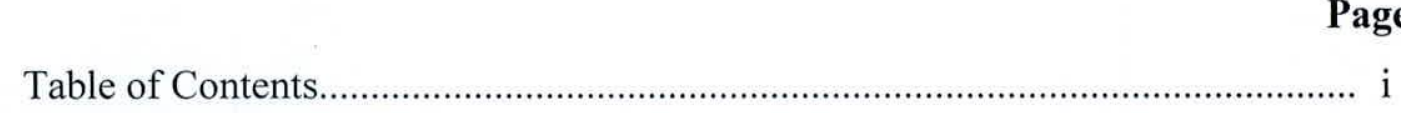

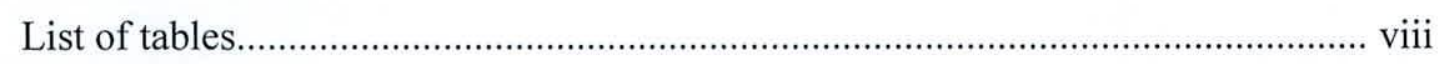

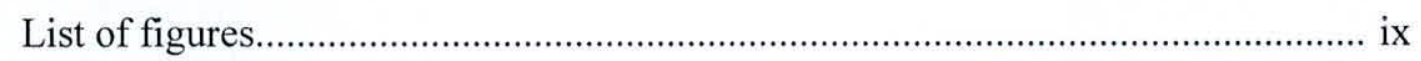

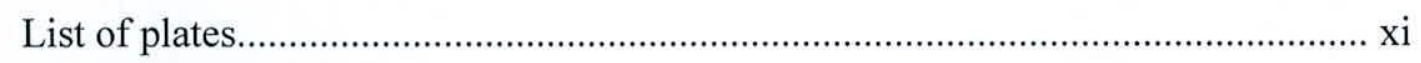

List of abbreviations.....................................................................................................

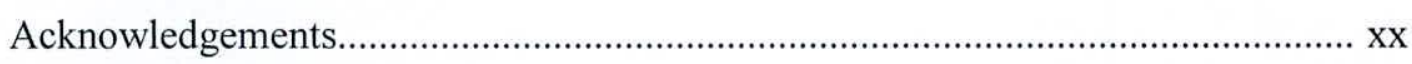

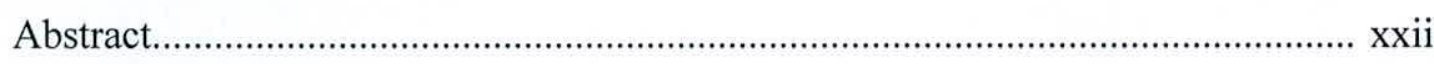

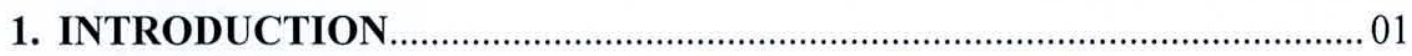

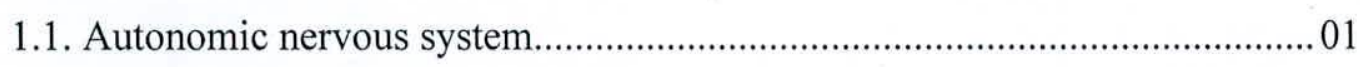

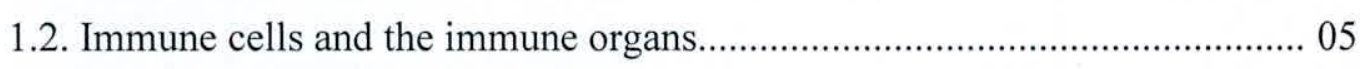

1.3. Autonomic innervation of lymphoid tissues.................................................. 06

1.5. Cholinergic innervation of localized inflammatory response ....................... 11

2. LITERATURE REVIEW .................................................................. 14

2.1. The cholinergic nervous system............................................................ 14

2.1.1. Acetylcholine .................................................................... 14

2.1.2. Receptors of the cholinergic system............................................ 17

2.1.2.1. Nicotinic acetylcholine receptors.................................. 18

2.1.2.2. Muscarinic acetylcholine receptors.............................. 19

2.2. General introduction to immune system................................................ 20

2.2.1. Infrastructure of immune system.......................................... 21

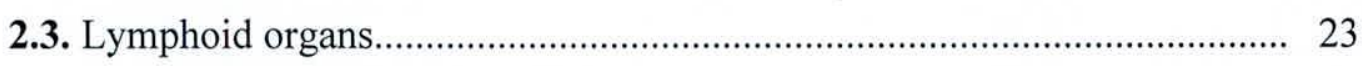

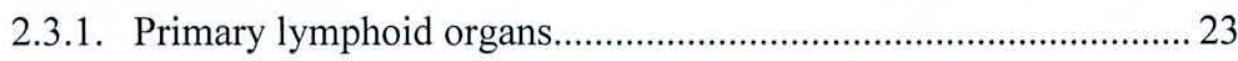

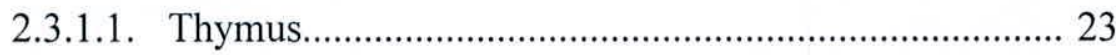

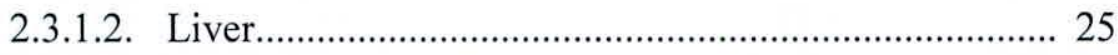

2.3.2. Secondary lymphoid organs..................................................... 26

2.3.2.1. Lymph node.................................................................... 26

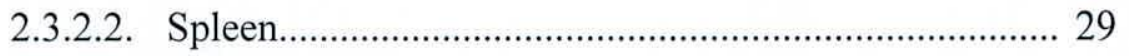

2.3.2.3. Mucosa associated lymphoid tissues............................ 31

2.4. Expression of $n A C h R s$ in lymphocytes and lymphoid tissues.................. 33

2.5. Expression of nAChRs in lymphocytes and lymphoid tissues.................. 34 
2.6. Autonomic innervation of the lymphoid tissues.

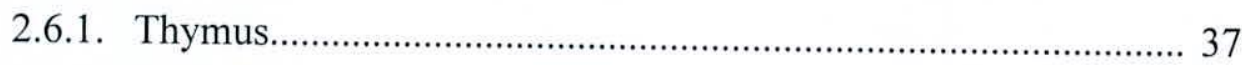

2.6.2. Spleen................................................................................. 38

2.6.3. Lymph node........................................................................ 40

2.6.4. Liver.................................................................................. 42

2.6.5. Peyer's patches..................................................................... 43

2.7. Non neuronal cholinergic systems............................................................ 44

2.8. Neuroimmune modulations.................................................................... 45

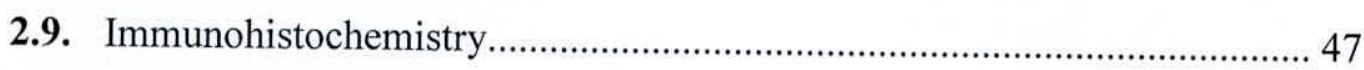

2.10. Immunoflourescence....................................................................... 54

2.11. Biomarkers of the cholinergic nervous system....................................... 57

2.12. Objectives..................................................................................... 59

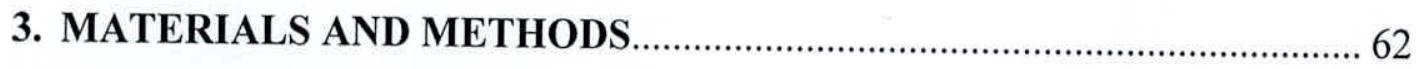

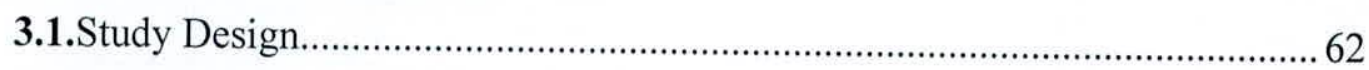

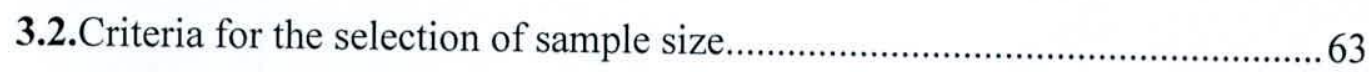

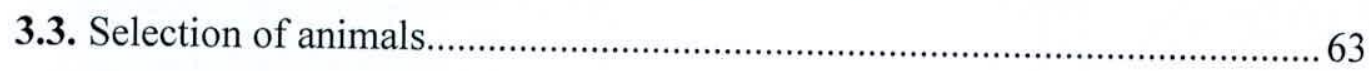

3.3.1. Balb/C mice........................................................................... 63

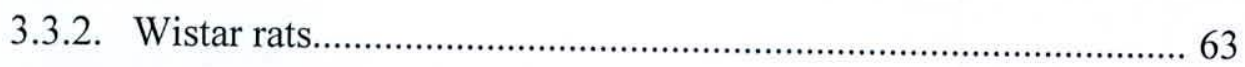

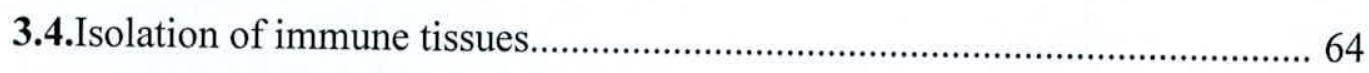

3.4.1. Collection of immune tissues of Balb/C mice...............................64 64

3.4.2. Collection of immune tissues of Wistar rats................................. 66

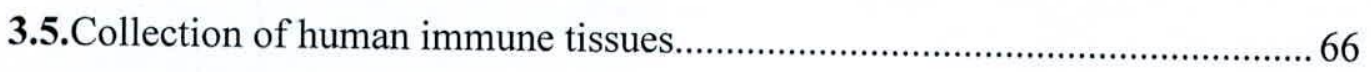

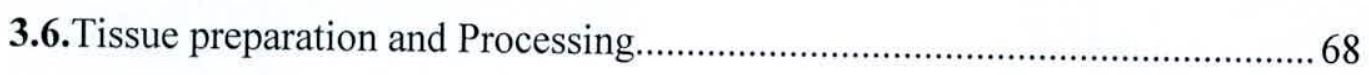

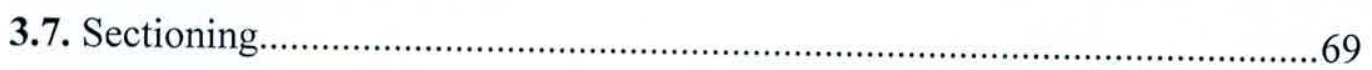

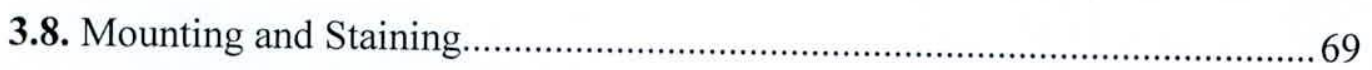

3.9. Haematoxylin \& Eosin staining procedure................................................. 70

3.10. Immunohistochemistry technique.............................................................. 71

3.10.1. Preparation of tissues.................................................................. 71

3.10.2. Removal of Paraffin and Rehydration..................................... 71

3.10.3. Heat induced antigen retrieval................................................. 72

3.10.4. Blocking of endogenous peroxidase activity.............................. 72

3.10.5. Blocking of tissues by Bovine Serum Albumin........................... 73

3.10.6. Primary Antibody Reaction........................................................ 73 
3.10.7. Secondary Antibody Reaction......................................................... 73

3.10.7.1. Selection of secondary antibody .................... 73

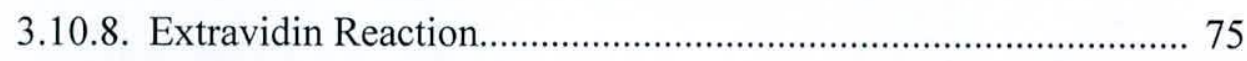

3.10.9. DAB Colour Reaction............................................................... 76

3.10.10. Clearing and Mounting............................................................. 76

3.11. Image collection and processing............................................................ 77

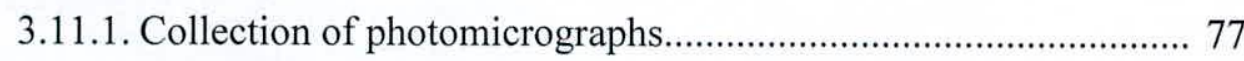

3.11.2. Processing of images................................................................... 80

3.11.2.1. Tissue permeabilization of primary antibodies............ 80

3.11.2.2. Evaluation of the immuno-stained images.................... 80

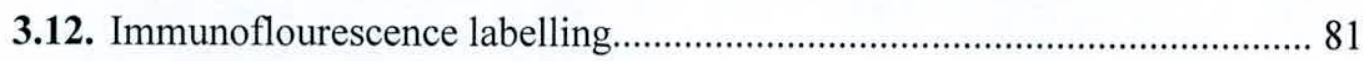

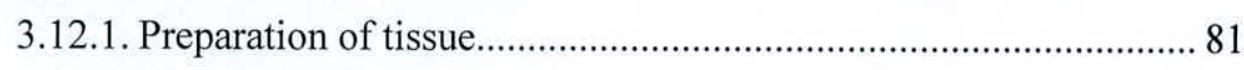

3.12.2. Addition of primary antibody ...................................................... 81

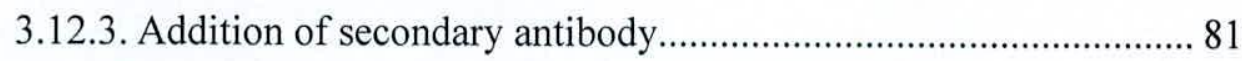

3.12.4. Mounting of immunoflourescence slides...................................... 82

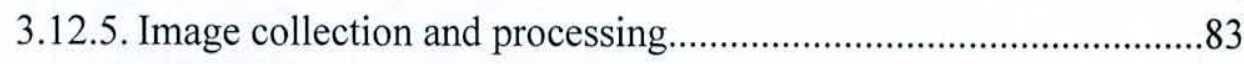

3.12.6. Detection of immunoflourescent signals....................................... 84

3.13. Induction of localized sterile inflammatory response................................. 86

3.13.1. Sterile turpentine administration................................................ 86

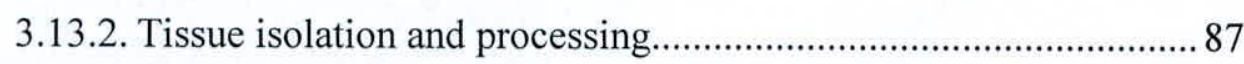

3.14. Immunohistochemistry of turpentine administered tissues.......................... 88

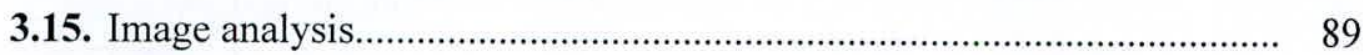

4. RESULTS

4.1. Micro structural variations of the lymphoid tissues.......................................... 90

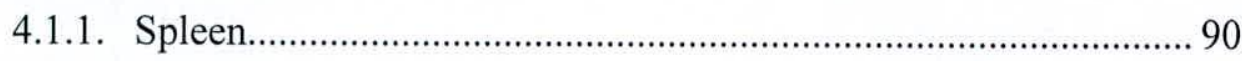

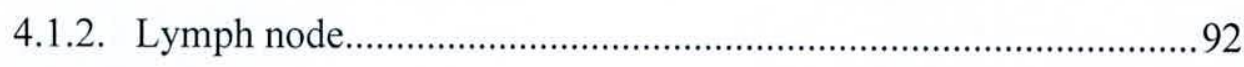

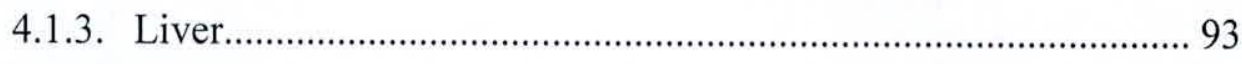

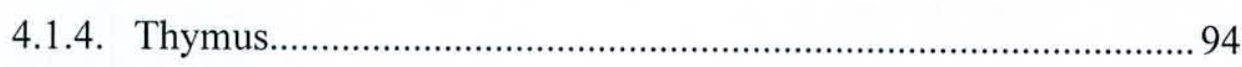

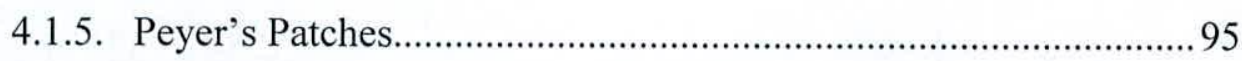

4.2.Distribution of $\alpha 1$ subunit of nicotinic acetylcholine receptors in immune tissues of Balb/C mice and humans ............................................................. 96

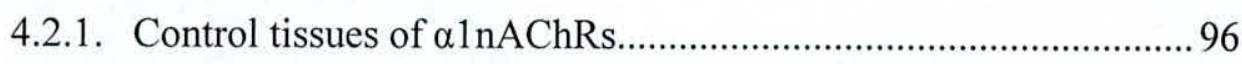

4.2.2. Distribution of $\alpha 1 \mathrm{nAChRs}$ in spleen of Balb/C mice................... 97 
4.2.3. Distribution of $\alpha \operatorname{lnAChRs~in~Lymph~node~of~Balb/C~mice........~} 98$

4.2.4. Distribution of $\alpha 1 \mathrm{nAChRs}$ in Thymus of Balb/C mice............. 99

4.2.5. Distribution of $\alpha 1 n A C h R s$ in Peyer's Patches of Balb/C mice.. 101

4.2.6. Distribution of $\alpha 1 \mathrm{nAChRs}$ in Liver of Balb/C mice...................102

4.2.7. Distribution of $\alpha \ln A C h R s$ in spleen of humans....................... 103

4.2.8. Distribution of $\alpha 1 n A C h R s$ in lymph node of humans .............. 103

4.2.9. Distribution of $\alpha \ln A C h R s$ in liver of humans............................ 104

4.2.10. Distribution of $\alpha \ln A C h R s$ in lymphoid aggregations of posterior part of tongue of humans 105

4.3. Distribution of $\alpha 7$ subunit of nicotinic acetylcholine receptors in immune tissues of Wistar rats mice and humans 106

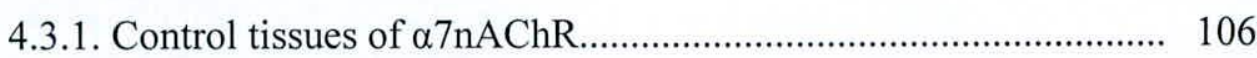

4.3.2. Distribution of $\alpha 7 \mathrm{nAChRs}$ in spleen of Wistar rats....................... 107

4.3.3. Distribution of $\alpha 7 \mathrm{nAChRs}$ in lymph node of Wistar rats............... 108

4.3.4. Distribution of $\alpha 7 \mathrm{nAChRs}$ in thymus of Wistar rats..................... 108

4.3.5. Distribution of $\alpha 7 \mathrm{nAChRs}$ in Peyer's patches of Wistar rats......... 109

4.3.6. Distribution of $\alpha 7 \mathrm{nAChRs}$ in liver of Wistar rats........................... 110

4.3.7. Distribution of $\alpha 7 \mathrm{nAChRs}$ in spleen of humans............................ 111

4.3.8. Distribution of $\alpha 7 \mathrm{nAChRs}$ in lymph node of humans.................... 111

4.3.9. Distribution of $\alpha 7 \mathrm{nAChRs}$ in Peyer's patches of humans.............. 112

4.3.10. Distribution of $\alpha 7 \mathrm{nAChRs}$ in liver of humans............................... 113

4.3.11. Distribution of $\alpha 7 \mathrm{nAChRs}$ in lymphoid aggregations of posterior part of tongue.

4.4. Distribution of M2 subunit of muscarinic acetylcholine receptors in immune tissues of Balb/C mice and Wistar rats. 114

4.4.1. Control tissues of M2AChRs..................................................... 114

4.4.2. Distribution of M2AChRs in spleen of Balb/C mice.................... 115

4.4.3. Distribution of M2AChRs in lymph node of Balb/C mice......... 115

4.4.4. Distribution of M2AChRs in thymus of Balb/C mice.................. 116

4.4.5. Distribution of $\mathrm{M} 2 \mathrm{AChRs}$ in liver of Balb/C mice....................... 117

4.4.6. Distribution of M2AChRs in Peyer's Patches of Balb/C mice...... 117

4.4.7. Distribution of $\mathrm{M} 2 \mathrm{AChRs}$ in spleen of Wistar rats...................... 118

4.4.8. Distribution of M2AChRs in lymph nodes of Wistar rats............ 118 
4.5. Distribution of M3 subunit of muscarinic acetylcholine receptors in immune tissues of humans

4.5.1. Control tissues of M3AChRs. 120

4.5.2. Distribution of M3 subtype of mAChRs in spleen of humans. 121

4.5.3. Distribution of M3 subtype of mAChRs in lymph nodes of human .. 121

4.5.4. Distribution of M3AChRs in Peyers Patches of human. 122

4.5.5. Distribution of M3AChRs in liver of human 123

4.6. Localization of Vesicular Acetylcholine Transporter Proteins in immune tissues of Balb/C mice, Wistar rats and humans. 124

4.6.1. Control tissues of VAChT 124

4.6.2. Localization of VAChT in spleen of Balb/C mice. 125

4.6.3. Localization of VAChT in lymph node of Balb/C mice. 125

4.6.4. Localization of VAChT in thymus of Balb/C mice. 126

4.6.5. Localization of VAChT in Peyer's patches of Balb/C mice. 127

4.6.6. Localization of VAChT in liver of Balb/C mice. 127

4.6.7. Localization of VAChT in spleen of Wistar rat 128

4.6.8. Localization of VAChT in lymph node of Wistar rats 128

4.6.9. Localization of VAChT in thymus of Wistar rats. 129

4.6.10. Localization of VAChT in Peyer's patches of Wistar rats. 129

4.6.11. Localization of VAChT in liver of Wistar rats. 130

4.6.12. Localization of VAChT in spleen of humans 130

4.6.13. Localization of VAChT in lymph node of humans . .131

4.6.14. Localization of VAChT in lymph node of humans. 131

4.7. Localization of Choline acetyltransferase enzyme activity in immune tissues of $\mathrm{Balb} / \mathrm{C}$ mice and Wistar rats 132

4.7.1. Control tissues of ChAT. 132

4.7.2. Localization of ChAT enzyme activity in spleen of Balb/C mice....... 133

4.7.3. Localization of ChAT enzyme in lymph node of Balb/C mice............133

4.7.4. Localization of ChAT enzyme activity in thymus of Balb/C mice......134

4.7.5. Localization of ChAT in Peyer's patches of Balb/C mice. 135

4.7.6. Localization of ChAT enzyme activity in liver of Balb/C mice.... 135 
4.7.7. Localization of ChAT enzyme activity in spleen of Wistar rats. 136

4.7.8. Localization of ChAT in lymph node of Wistar rats.......................... 136

4.7.9. Localization of ChAT enzyme activity in thymus of Wistar rats........ 137

4.7.10. Localization of ChAT in Peyer's patches of Wistar rats................... 138

4.7.11. Localization of ChAT in liver of Wistar rats................................... 138

4.8. Localization of the Neuron Specific Enolase enzyme activity in immune tissues of human. 139

4.8.1. Control tissues. 139

4.8.2. Localization of NSE enzyme activity in spleen of humans 140

4.8.3. Localization of NSE enzyme activity in lymph nodes of humans.......140

4.8.4. Localization of NSE enzyme activity in Peyer's patches of humans... 141

4.8.5. Localization of NSE enzyme activity in liver of humans 141

4.9. Localization and identification of cholinergic components in the immune tissues of Balb/C mice, Wistar rats and human using immunoflourescence detection technique. 143

4.9.1. Localization of the $\alpha 1 n A C h R s$ in the immune tissues of humans ..... 143

4.9.2. Localization of the $\alpha 7 \mathrm{nAChRs}$ in the immune tissues of humans...... 144

4.9.3. Localization of the VAChT in the immune tissues of humans.........145

4.9.4. Localization of the NSE in the immune tissues of humans. 146

4.10. Comparison of the results produced regarding the localization and distribution of cholinergic receptors and their nerve endings by immunohistochemistry and immunoflourescence techniques.

4.10.1. The photomicrographs of IHC and IF staining of $\alpha 1 \mathrm{nAChRs}$ in human tissues to show the similar expression

4.10.2. The photomicrographs of IHC and IF staining of $\alpha 1 \mathrm{nAChRs}$ in human immune tissues to show the similar expression.

4.10.3. The photomicrographs of IHC and IF staining of VAChTin human immune tissues to show the similar expression.

4.10.4. The photomicrographs of IHC and IF staining of ChAT in immune tissues of Wistar rats to show the similar expression.

4.10.5. The photomicrographs of IHC and IF staining of NSE in human immune tissues to show the similar expression.

4.11. Histopathology of sterile abscesses 
4.12. Distribution of $\alpha 1 \mathrm{nAChRs}$ in sterile abscesses...........................................155

4.13. Localization of VAChTs in sterile abscesses............................................ 157

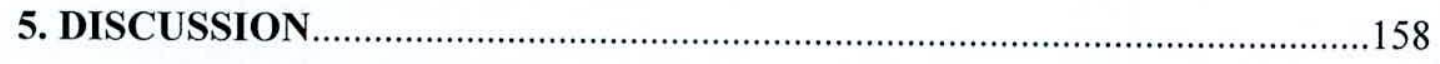

5.1. Localization and distribution of cholinergic components in thymus.......... 159

5.2 .

Localization and distribution of cholinergic components in spleen.

5.3. Localization and distribution of cholinergic components in lymph node... 168

5.4. Localization and distribution of cholinergic components in liver. 170

5.5. Localization \& distribution of cholinergic components in Peyer's patches. 172

5.6. Advantages of using different immunodetection techniques.

5.7. Cholinergic control of localized inflammatory response. 176

5.8. Limitations of the study.

5.9. Future recommendations.

6. CONCLUSIONS.

7. REFERENCES.

8. Appendices.

Appendix 1 - List of Publications and Communications. 218

Appendix 2a - Ethical Clearance of animal study. 222

Appendix $2 b$ - Ethical Clearance of human study. 223

Appendix 3 - Consent form. 224

Appendix 4 - Preparation of reagents. 226 


\section{LIST OF TABLES}

Page

Table 3.1 - Primary antibodies used, their dilutions and positive control tissues..... 74

Table 3.2 - Secondary antibodies used and their dilution. 75

Table 3.3 - Different tissues used as positive controls in the manufacturers' Catalogues. .78

Table 3.4- Intensity of IHC immunostaining for the application of AChRs and biomarkers of cholinergic nerve endings.

Table 3.5 - Primary antibodies used, dilutions, positive control tissues in IF 82

Table 3.6 - Secondary antibodies used and their dilutions in IF.... .83

Table 3.7- Photomicrographs of tissues used as positive controls $\&$ stained with IF in the manufacturers' catalogue. .85

Table 4.1- Summarised immunoreactive values of the intensity of cholinergic receptors; $\alpha 1, \alpha 7, \mathrm{M} 2, \mathrm{M} 3, \mathrm{VAChT}$, ChAT and NSE in immune tissues of Balb/C mice, Wistar rats and humans. 


\section{LIST OF FIGURES}

Figure 2.1 Biochemical reactions take place in the cholinergic nerve ending.......58

Figure 2.2 Topology of single receptor subunit in the cell membrane....................20

Figure 2.3 Diagrammatic representation of thymic microstructure........................25

Figure 2.4 Diagrammatic representation of the microstructure of

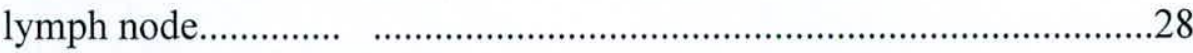

Figure 2.5 Diagrammatic representation of the microstructure of spleen...............31

Figure 2.6 Diagrammatic representation of LSAB method of IHC.........................55

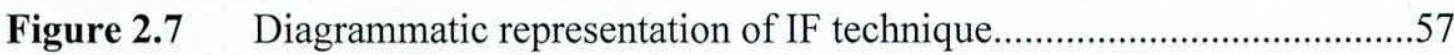

Figure 3.1 Diagrammatic representation of a dissected mouse ..............................65

Figure 3.2 Positively stained image of immunoperoxidase staining of monoclonal $\alpha \ln A C h R$ as primary antibody

Figure 3.3 Positively stained image of immunoperoxidase staining of monoclonal $\alpha 7 \mathrm{nAChR}$ as primary antibody

Figure 3.4 Positively stained image of immunoperoxidase staining of polyclonal anti- Vesicular Acetylcholine Transporter .78

Figure 3.5 Positively stained image of immunoperoxidase staining of polyclonal Anti-choline acetyl transferase as primary antibody.....

Figure 3.6 Positively stained image of immunoperoxidase staining of polyclonal anti-M2AChR as primary antibody.

Figure 3.7 Positively stained image of immunoperoxidase staining of polyclonal anti-M3AChR as primary antibody....

Figure 3.8 Positively stained image of immunoperoxidase staining of Polyclonal anti-Neuron specific enolase as primary antibody

Figure 3.9 Positively stained image of Immuofluorescent staining of $\alpha 7 \mathrm{nAChR}$ in the rat cerebellum.

Figure 3.10 Positively stained image of Immuofluorescent staining of $\mathrm{ChAT}$ in the rat brain section.

Figure 3.11 Positively stained image of Immuofluorescent staining of VAChT in isolated cells. 
Figure 5.1 Diagrammatic representation of the suggested distribution of AChRs and the cholinergic innervation pattern of thymus referring to the immunoreactivity of AChRs, VAChT and ChAT enzyme activity.

Figure 5.2 Diagrammatic representation of the possible distribution of AChRs and the cholinergic innervation pattern of a spleen referring to the immunoreactivity of AChRs, VAChT, ChAT and NSE.

Figure 5.3 Diagrammatic representation of the possible distribution of AChRs and the cholinergic innervation pattern of a lymph node referring to the immunoreactivity of AChRs, VAChT, ChAT and NSE 


\section{LIST OF PLATES}

Plate 4.1 Photomicrographs displaying the microscopic features in paraffin embedded sections of spleens of Balb/C mice, Wistar rats and human in $\mathrm{H} \& \mathrm{E}$ staining.....

Plate 4.2 Photomicrographs displaying the microscopic features in the paraffin embedded sections of lymph nodes of Balb/C mice, Wistar rats and human in $\mathrm{H} \& \mathrm{E}$ staining. 92

Plate 4.3 Photomicrographs displaying the microscopic features in paraffin embedded sections of liver of $\mathrm{Balb} / \mathrm{C}$ mice, Wistar rats and human in $\mathrm{H}$ \& $\mathrm{E}$ staining.

Plate 4.4 Photomicrographs displaying the microscopic features in the paraffin embedded sections of thymus of Balb/C mice and Wistar rats in $\mathrm{H} \& \mathrm{E}$ staining..94

Plate 4.5 Photomicrographs displaying the microscopic features in paraffin embedded sections of Peyer's patches of Balb/C mice and Wistar rats in $\mathrm{H} \& \mathrm{E}$ staining.

Plate 4.6 Photomicrographs displaying the immunohistochemical staining of $\alpha 1 n A C h R s$ in paraffin embedded sections of positive and negative control tissues of primary antibody

Plate 4.7 Photomicrographs displaying the immunohistochemical staining of $\alpha \operatorname{lnAChRs}$ in paraffin embedded sections of spleen of Balb/C mice.

Plate 4.8 Photomicrographs displaying the immunohistochemical staining of $\alpha \operatorname{lnAChRs}$ in paraffin embedded sections of lymph node of Balb/C mice.

Plate 4.9 Photomicrographs displaying the immunohistochemical staining of $\alpha 1 \mathrm{nAChRs}$ in paraffin embedded sections of thymus of Balb/C mice 100

Plate 4.10 Photomicrographs displaying the immunohistochemical staining of $\alpha \operatorname{lnAChRs}$ in paraffin embedded sections of Peyer's Patches of Balb/C mice. 101

Plate 4.11 Photomicrograph displaying the immunohistochemical staining of $\alpha 1 \mathrm{nAChRs}$ in paraffin embedded sections of Liver of Balb/C mice. 102

Plate 4.12 Photomicrographs displaying the immunohistochemical staining of $\alpha 1 \mathrm{nAChRs}$ in paraffin embedded sections of spleen of human tissues. 103

Plate 4.13 Photomicrographs displaying the immunohistochemical staining of $\alpha \ln A C h R s$ in paraffin embedded sections of human lymph node. 104 
Plate 4.14 Photomicrographs displaying the immunohistochemical staining of $\alpha 1 \mathrm{nAChRs}$ in paraffin embedded sections of human liver tissue

Plate 4.15. Photomicrographs displaying the immunohistochemical staining of $\alpha \operatorname{lnAChRs}$ in paraffin embedded section of the lymphoid aggregations of human tongue. 105

Plate 4.16 Photomicrographs displaying the immunohistochemical staining of $\alpha 7 \mathrm{nAChRs}$ in paraffin embedded sections of human cerebral cortex.

Plate 4.17 Photomicrographs displaying the immunohistochemical staining of $\alpha 7 \mathrm{nAChRs}$ in paraffin embedded sections of spleen of Wistar rats. 107

Plate 4.18 Photomicrographs displaying the immunohistochemical staining of $\alpha 7 \mathrm{nAChRs}$ in paraffin embedded sections of lymph node of Wistar rats. 108

Plate 4.19 Photomicrographs displaying the immunohistochemical staining of $\alpha 7 \mathrm{nAChRs}$ in paraffin embedded sections of thymus of Wistar rats. 109

Plate 4.20 Photomicrographs displaying the immunohistochemical staining of $\alpha 7 \mathrm{nAChRs}$ in paraffin embedded sections of Peyer's patches of Wistar rats..... 110

Plate 4.21 Photomicrograph displaying the immunohistochemical staining of $\alpha 7 \mathrm{nAChRs}$ in paraffin embedded sections of liver of Wistar rats. 110

Plate 4.22 Photomicrographs displaying the immunohistochemical staining of $\alpha 7 \mathrm{nAChRs}$ in paraffin embedded sections of human spleen 111

Plate 4.23 Photomicrographs displaying the immunohistochemical staining of $\alpha 7 \mathrm{nAChRs}$ in paraffin embedded sections of lymph nodes of humans. 112

Plate 4.24 Photomicrograph displaying the immunohistochemical staining of $\alpha 7 \mathrm{nAChRs}$ in paraffin embedded sections of Peyer's patches of humans. 112

Plate 4.25 Photomicrograph displaying the immunohistochemical staining of $\alpha 7 \mathrm{nAChRs}$ in paraffin embedded sections of liver of human 113

Plate 4.26 Photomicrograph displaying the immunohistochemical staining of $\alpha 7 \mathrm{nAChRs}$ in paraffin embedded sections of lymphoid aggregations of posterior tongue of human

Plate 4.27 Photomicrograph displaying the immunohistochemical staining of M2AChRs in paraffin embedded sections of cerebral cortex of Wistar rats

Plate 4.28 Photomicrographs displaying the immunohistochemical staining of M2AChRs in paraffin embedded sections of spleen of Balb/C mice. 115

Plate 4.29 Photomicrographs displaying the immunohistochemical staining of $\mathrm{M} 2 \mathrm{AChRs}$ in paraffin embedded sections of lymph node of Balb/C mice..... .116 
Plate 4.30 Photomicrographs displaying the immunohistochemical staining of M2AChRs in paraffin embedded sections of thymus of Balb/C mice.

Plate 4.31 Photomicrograph displaying the immunohistochemical staining of $\mathrm{M} 2 \mathrm{AChRs}$ in paraffin embedded sections of liver of Balb/C mice 117

Plate 4.32 Photomicrograph displaying the immunohistochemical staining of $\mathrm{M} 2 \mathrm{AChRs}$ in paraffin embedded sections of Peyer's patches recovered from Balb/C mice.

Plate 4.33 Photomicrographs displaying the immunohistochemical staining of $\mathrm{M} 2 \mathrm{AChRs}$ in paraffin embedded sections of spleen of Wistar rats 118

Plate 4.34 Photomicrographs displaying the immunohistochemical staining of M2AChRs in paraffin embedded sections of lymph node of Wistar rat.

Plate 4.35 Photomicrograph displaying the immunohistochemical staining of M2AChRs in paraffin embedded sections of thymus recovered from Wistar rats.119

Plate 4.36 Photomicrograph displaying the immunohistochemical staining of M3AChRs in paraffin embedded sections of small intestinal wall of human tissue used as positive control.

Plate 4.37 Photomicrograph displaying the immunohistochemical staining of M3AChRs in paraffin embedded sections of spleen of human tissue.

Plate 4.38 Photomicrograph displaying the immunohistochemical staining of M3AChRs in paraffin embedded sections of lymph node.

Plate 4.39 Photomicrograph displaying the immunohistochemical staining of M3AChRs in paraffin embedded sections of small intestinal wall of human tissue..122

Plate 4.40 Photomicrograph displaying the immunohistochemical staining of M3AChRs in paraffin embedded sections of liver of human tissue.....

Plate 4.41 Photomicrograph displaying the immunohistochemical staining of VAChT in paraffin embedded sections of skeletal muscle tissue of human used as positive control.

Plate 4.42 Photomicrographs displaying the immunohistochemical staining of $\mathrm{VAChT}$ in paraffin embedded sections of spleen of Balb/C mice.

Plate 4.43 Photomicrographs displaying the immunohistochemical staining of VAChT in paraffin embedded sections of lymph node of Balb/C mice.

Plate 4.44 Photomicrographs displaying the immunohistochemical staining of VAChT in paraffin embedded sections of thymus of Balb/C mice

Plate 4.45 Photomicrograph displaying the immunohistochemical staining of VAChT in paraffin embedded sections of Peyer's patches of Balb/C mice 
Plate 4.46 Photomicrograph displaying the immunohistochemical staining of

VAChT in paraffin embedded sections of liver of Balb/C mice.....

Plate 4.47 Photomicrograph displaying the immunohistochemical staining of VAChT in paraffin embedded sections of spleen of Wistar rats

Plate 4.48 Photomicrograph displaying the immunohistochemical staining of VAChT in paraffin embedded sections of lymph node of Wista rats.

Plate 4.49 Photomicrograph displaying the immunohistochemical staining of VAChT in paraffin embedded sections of thymus of Wistar rats.

Plate 4.50 Photomicrograph displaying the immunohistochemical staining of VAChT in paraffin embedded sections of Peyer's patches of Wistar rats....

Plate 4.51 Photomicrograph displaying the immunohistochemical staining of VAChT in paraffin embedded sections of liver of Wistar rat.

Plate 4.52 Photomicrograph displaying the immunohistochemical staining of VAChT in paraffin embedded sections of spleen of human

Plate 4.53 Photomicrograph displaying the immunohistochemical staining of VAChT in paraffin embedded sections of lymph node of human.

Plate 4.54 Photomicrograph displaying the immunohistochemical staining of VAChT in paraffin embedded sections of Peyer's patches of human tissues.

Plate 4.55 Photomicrograph displaying the immunohistochemical staining of ChAT in paraffin embedded sections of cerebral cortex of Wistar rats shown as the positive control

Plate 4.56 Photomicrograph displaying the immunohistochemical staining of ChAT in paraffin embedded sections of spleen of Balb/C mice.

Plate 4.57 Photomicrograph displaying the immunohistochemical staining of ChAT in paraffin embedded sections of lymph node of Balb/C mice

Plate 4.58 Photomicrographs displaying the immunohistochemical staining of ChAT in paraffin embedded sections of thymus of Balb/C mice.....

Plate 4.59 Photomicrograph displaying the immunohistochemical staining of ChAT in paraffin embedded sections of Peyer's patches of Balb/C mice.

Plate 4.60 Photomicrograph displaying the immunohistochemical staining of ChAT in paraffin embedded sections of liver of Balb/C mice.....

Plate 4.61 Photomicrographs displaying the immunohistochemical staining of ChAT in paraffin embedded sections of spleen of Wistar rats..... 136

Plate 4.62 Photomicrograph displaying the immunohistochemical staining of ChAT in paraffin embedded sections of lymph node of Wistar rats. 
Plate 4.63 Photomicrograph displaying the immunohistochemical staining of ChAT in paraffin embedded sections of thymus of Wistar rats.

Plate 4.64 Photomicrograph displaying the immunohistochemical staining of ChAT in paraffin embedded sections of Peyer's patches of Wistar rats. 138

Plate 4.65 Photomicrograph displaying the immunohistochemical staining of ChAT in paraffin embedded sections of liver of Wistar rats. 138

Plate 4.66 Photomicrograph displaying the immunohistochemical staining of NSE in paraffin embedded sections of cerebral cortex of human tissue used as positive control

Plate 4.67 Photomicrograph displaying the immunohistochemical staining of NSE in paraffin embedded sections of spleen of human.

Plate 4.68 Photomicrograph displaying the immunohistochemical staining of NSE in paraffin embedded sections of lymph node of human.

Plate 4.69 Photomicrograph displaying the immunohistochemical staining of NSE in paraffin embedded sections of Peyer's patches of human

Plate 4.70 Photomicrograph displaying the immunohistochemical staining of NSE in paraffin embedded sections of liver of human

Plate 4.71 Single labelling immunoflourescence (IF) staining of $\alpha \ln A C h R$ in paraffin embedded sections of immune tissues of humans.

Plate 4.72 Single labelling immunoflourescence staining of $\alpha 7 \mathrm{nAChR}$ in paraffin embedded sections of immune tissues of humans.

Plate 4.73 Single labelling immunoflourescence staining of VAChT in paraffin embedded sections of immune tissues of humans.

Plate 4.74 Single labelling immunoflourescence staining of NSE in paraffin embedded sections of immune tissues of humans............................................. 146

Plate 4.75 Lymph node of human displaying the immunohistochemical staining and immunoflourescence staining of $\alpha \ln A C h R$. 148

Plate 4.76 Lymph node of human displaying the immunohistochemical staining and immunoflourescence staining of $\alpha \ln \mathrm{AChR}$.

Plate 4.77 Spleen of human displaying the immunohistochemical and immunoflourescence staining of $\alpha 7 \mathrm{nAChR}$

Plate 4.78 Spleen of human displaying the immunohistochemical and immunoflourescence staining of $\alpha 7 \mathrm{nAChR}$

Plate 4.79 Liver of human displaying the immunohistochemical and immunoflourescence staining of $\alpha 7 \mathrm{nAChR}$ 
Plate 4.80 Spleen of human displaying the immunohistochemical and immunoflourescence staining of VAChT

Plate 4.81 Lymph node of human displaying the immunohistochemical and immunoflourescence staining of VAChT.

Plate 4.82 Spleen of human displaying the immunohistochemical and immunoflourescence staining of ChAT ........................................................... 151

Plate 4.83 Thymus of human displaying the immunohistochemical and immunoflourescence staining of ChAT...

Plate 4.84 Spleen of human displaying the immunohistochemical and immunoflourescence staining of NSE.

Plate 4.85 Lymph node of human displaying the immunohistochemical and immunoflourescence staining of NSE

Plate 4.86 Liver of human displaying the immunohistochemical and immunoflourescence staining of NSE 153

Plate 4.87 Sterile turpentine injected abscess tissue and the control mice tissue stained with Haematoxylin \& Eosin.

Plate 4.88 Immunohistochemical identification of $\alpha \ln A C h R$ in paraffin embedded sections of abscess tissue of Balb/C mice. 156

Plate 4.89 Immunohistochemical identification of VAChT in paraffin embedded sections of abscess tissue of Balb/C mice. 


\section{List of Abbreviations}

\begin{tabular}{|c|c|}
\hline $\mathrm{Ab}$ & Antibody \\
\hline $\mathrm{ABC}$ & Avidin biotin complex method \\
\hline $\mathrm{ACh}$ & Acetylcholine \\
\hline $\mathrm{AChE}$ & Acetylcholine esterase \\
\hline $\mathrm{AChR}$ & Acetylcholine receptor \\
\hline $\mathrm{Ag}$ & Antigen \\
\hline ANS & Autonomic nervous system \\
\hline APC & Antigen presenting cells \\
\hline AR & Antigen retreival \\
\hline BALT & Bronchial mucosa associated lymphoid tissue \\
\hline $\mathrm{BChE}$ & Butyrylcholine esterase \\
\hline BSA & Bovine serum albumin \\
\hline cAMP & Cyclic AMP \\
\hline CAP & Cholinergic anti- inflammatory pathway \\
\hline CGRP & Calcitonin gene related peptide \\
\hline ChAT & Choline acetyl transferase \\
\hline CHT & Choline transporter \\
\hline CNS & Central nervous system \\
\hline $\mathrm{AB}$ & Diamino benzedene \\
\hline $\mathrm{BH}$ & Dopamine betahydroxilase \\
\hline ENS & Enteric nervous system \\
\hline FDCs & Follicular dendritic cells \\
\hline ITC & Fluorescein isothiocyanate \\
\hline I \& E & Heamatoxillin and Eosin \\
\hline ? & Hydrogen peroxide \\
\hline
\end{tabular}




\begin{tabular}{ll} 
HEVs & High endothelial venules \\
HIER & Heat induced epitope retreival \\
IgG & Immunoglobulin G \\
IHC & Immunohistochemistry \\
IF & Immunoflourescence \\
IL 2 & Interleukin 2 \\
INF-c & Inteferon c \\
IR & Immunoreactivity \\
LSAB & Labelled streptavidin biotin method \\
M2AChR & M2 subunit of muscarinic acetylcholine receptor \\
M3AChR & M3 subunit of muscarinic acetylcholine receptor \\
mAChR & muscarinic acetylcholine receptor \\
MALT & Mucosa associated lymphoid tissue \\
MHC & Major histocompatibility complex \\
MNLs & Mononuclear leukocytes \\
nAChR & nicotinic acetylcholine receptor \\
NALT & Nasopharynx associated lymphoid tissue \\
NPY & Neuropeptide Y \\
NSE & Neuron specific enolase \\
PAL & Peri-arteriolar lymphoid sheath \\
PBS & Phosphate buffered saline \\
PGP & Protein gene product \\
PL & Polymorpho leukocytes \\
PLC & Phospholipase C \\
PNS & Periphral nervous system \\
\hline Peyer's patches \\
PP
\end{tabular}


PRV Pseudorabies virus

SP Substance P

TH Tyrosine hydroxilase

TNF Tumour necrosis factor

TRITC Tetramethyl rhodamine isothiocyanate

VAChT Vesicular acetylcholine transporter

VIP Vasoactive intestinal polypeptide

WGA-HRP Wheat germ agglutinin conjugated horseradish peroxidase

$\alpha \operatorname{lnAChR} \quad \alpha 1$ subunit of nicotinic acetylcholine receptor

$\alpha 7 \mathrm{nAChR} \quad \alpha 7$ subunit of nicotinic acetylcholine receptor

M2AChR M2 subtype of muscarinic acetylcholine receptor

M3AChR M3 subtype of muscarinic acetylcholine receptor 


\section{Acknowledgements}

I wish to express my heartfelt and most sincere gratitude to my supervisor Prof. Surangi. G.Yasawardene, Professor of Anatomy and Head, Department of Anatomy, Faculty of Medical Sciences, University of Sri Jayewardenepura for her encouragement, excellent guidance, keen interest, fruitful discussions, parental advice and unending support to complete the research successfully. Dear Madam, it is indeed an honour to be your student, thank you again.

I am deeply indebted to the staff of the Department of Anatomy, especially for their unending support and assistance given to complete my research successfully.

I greatly acknowledge all my colleagues for their excellent support and willingness to help in many ways to conduct this research.

I greatly acknowledge Prof. Lakmini Mudduwa, Professor in Pathology, Department of Pathology, Faculty of Medicine, University of Ruhunu, for her guidance in reading the immunohistochemistry findings produced in this research. Also I am greatly indebted for the valuable support given by her to perform the technique, as well as to master the skills in immunohistochemistry technique. I'm very much thankful to the former Head of the Department of Pathology, Faculty of Medicine, University of Ruhunu, late Dr. Ajith Lamahewage for his valuable support and Mrs. Dammika Gunawardhene, technical officer of the Department of Pathology for her support and willingness to help in learning the immunohistochemistry technique.

I wish to acknowledge the Coordinator and the technical officers of Animal house of Faculty of Medical Sciences, University of Sri Jayewardenepura, who gave permission to maintain and dissect laboratory animals. I also wish to thank the Veterinary Medical Officer and the staff of the animal house of Medical research Institute for providing $\mathrm{Balb} / \mathrm{C}$ mice and Wistar rats whenever I need them. 
I greatly acknowledge the Head and staff of the Department of Forensic Medicine and the staff of the JMO office of the Colombo South Teaching Hospital in collecting postmortem immune tissue samples. My sincere gratitude goes to the relatives of the deceased who gave consent to collect post-mortem samples.

I am very much thankful to the, Head and staff of the Department of Parasitology, Faculty of Medical Sciences, University of Sri Jayewardenepura for providing assistance in utilizing some of their instruments, for preparation photomicrographs and operating Olympus FSX 100. I am also thankful to the Head and the staff of the Department of Microbiology and Department of Pathology for providing assistance in performing the research.

I am grateful to my supervisor for the financial support through the Grants ASP/06/RE/2009/11 and ASP/06/Res/Med/2011/20 of University of Sri Jayewardenepura to carry out the research work uninterruptedly and the financial support of National Science Foundation, Sri Lanka of Grant no: RG/2011/HS/02.

I specially thank the University Grants Commission for the Grant no: UGC/ICD/CRF2009/1/6 which made the research to upgrade from M Phil in to PhD degree.

Finally I wish to acknowledge the former Head Dr. Rajendra Prasad and the present Head of the Department of Human Biology, the Dean of Faculty of Health- Care Sciences and the Vice Chancellor of Eastern University for granting study leave to finish my degree.

The thesis is dedicated to my parents, my husband and my two children. 


\section{Identification and localization of cholinergic nerve endings and}

\section{acetylcholine receptors in tissues of the immune system- an animal and \\ postmortem human study \\ Mythreye Thayabaran}

\section{ABSTRACT}

The central nervous system is known to interact with the immune system through the autonomic nerves and modify the immune responses. Although sympathetic noradrenergic fibres has been considered as the predominant component controlling the functions of lymphoid tissues, recent researches point towards equally important role of cholinergic innervation. Our present study describes the cholinergic nerve supply of different lymphoid tissues by demonstrating the neural profiles, cholinergic nerve endings, acetylcholine receptors and enzymes of acetylcholine synthesis by immunohistochemistry and immunoflourescence techniques.

Fresh immune tissues such as spleen, liver, lymph nodes, thymus and terminal ileum of small intestine were retrieved from $\mathrm{Balb} / \mathrm{C}$ mice, Wistar rats and humans and were processed to examine the microscopic ultra structural variations in Haematoxylin \& Eosin stained slides. Primary antibodies raised in rabbit and rat against $\alpha 1 \& \alpha$ 7subunits of nAChRs, M2 \& M3 subunits of mAChRs, VAChT, ChAT, and Neuron Specific Enolase (Sigma Aldrich, USA) were used to label the $4 \mu \mathrm{m}$ thin tissue sections. Biotinylated anti-rat and anti-rabbit IgG were used as secondary antibodies. Labelled Streptavidin Biotin (LSAB) technique was used; with DAB as chromogen and counterstained with Mayer's haematoxylin. Skeletal muscle and cerebral cortex were processed as positive controls. Similarly, immunoflourescence technique was done by labelling the tissues with the above mentioned primary antibodies and FITC conjugated secondary IgGs. Immunohistochemistry slides were observed and digitalized using 
Dino capture (Taiwan), while the immunoflourescence slides observed through advanced fluorescence microscope (Olympus FSX100). An inflammatory response was induced in Balb/C mice by injecting irritant sterile turpentine in their hind limbs and the histopathological changes examined in tissues presented with sterile abscess and local lymph nodes using $\mathrm{H} \& \mathrm{E}$ staining. Further using anti- $\alpha \ln \mathrm{AChR}$ and anti-VAChT in those tissues cholinergic innervation was investigated.

The immunohistochemistry slides were evaluated and the intensity of immunostaining was determined based upon a score of 0 (No staining), $1+$ (focal staining/ less than $30 \%$ of IR cells), $2+$ (focal to diffuse/moderate staining, 30\% - 60\% cells), 3+ (Strong staining, $61-100 \%$ of cells) (Schaulder et al, 2008).

Immunoreactivity of $\alpha 1 \& \alpha 7 \mathrm{nAChRs}$ and the M2AChRs were clearly identified in capsule, interlobar septa, corticomedullary junction and the cortex in thymus of Balb/C mice and Wistar rats. Similarly, VAChT and ChAT were localized at the proximities of those sites. In spleen the distribution of $\alpha 1 \& \alpha 7 n$ AChRs and the M2 \& M3 mAChRs were identified in the capsule, trabeculae and sinusoids of red pulp in the $\mathrm{Balb} / \mathrm{C}$ mice, Wistar rats and humans and the VAChT, ChAT and NSE were also localized towards their vicinity. The distribution of $\alpha 1 \& \alpha 7 \mathrm{nAChRs}$ and the M2 \& M3AChRs and the localization of VAChT and ChAT were mainly found in the capsule subcapsular sinus, medullary cords and paracortex of lymph nodes of the three species. Peyer's patches, liver and the lymphoid aggregations of posterior part of tongue did not express immunoreactivity towards anti-VAChTs, and ChAT. The distribution of $\alpha 7 \mathrm{nAChRs}$ and the M2 \& M3 mAChRs were not identified in those tissues. The expression of NSE at peri-portal triad of liver shows the nerve control of this tissue. It is apparent that the AChRs specifically localize in the sites rich in $\mathrm{T}$ cells. Further $\alpha 7 \mathrm{nAChRs}$ were found mainly in the subcellular compartments distributed with tissue macrophages. The strong 
IR of neuron specific enolase in capsules of spleen and lymph nodes provides evidence that the innervation from autonomic nerves traverse through their supporting framework reinforced by reticulin fibres to the parenchyma. The expression of $\alpha \ln A C h R$ in the polymorpho nuclear leukocyte and macrophages and the localization of VAChT in the similar sites confirm that parasympathetic cholinergic innervation in those sites and suggest that the inflammatory response may be regulated through $\alpha \ln A C h R s$. Localization of VAChT and ChAT confirms that the presence of cholinergic neural control of immune tissues is predominantly through the $\alpha 1 \& \alpha 7 \mathrm{nAChRs}$ and M2 \& M3 AChRs.

Knowledge gained regarding the in-situ localization and expression of cholinergic receptors could be used as a consistent anatomical background to understand the functional interaction between nervous and immune system leading to intervention of new therapeutic applications. The parasympathetic receptor distribution suggests cholinergic control of immune responses and by regulation of excess inflammatory response by the inflammatory reflex. A complete understanding of parasympathetic innervation of immune tissues is essential for designing more specific molecular based drug treatment in autoimmune and inflammatory diseases. 